\title{
La Universidad Nacional de Ingeniería (UNI) a través de sus patentes y modelos de utilidad Producción y análisis de redes
}

\section{(1) Carlos Enrique Agüero Aguilar}

Universidad Nacional Mayor de San Marcos I cagueroa@unmsm.edu.pe / https://orcid.org/oooo-o0o2-8254-230X

\section{Resumen}

El estudio hace visible las redes de colaboración en la producción de patentes y modelos de utilidad de la Universidad Nacional (UNI) de Ingeniería registradas en Espacenet hasta 2019. Se aplica el Análisis de Redes (AR) para medir el grado de colaboración de inventores y organizaciones, así como para determinar las principales líneas de interés tecnológico. Se identificaron 104 inventores, la colaboración entre 2 organizaciones y 65 líneas de interés tecnológico. Se observó un incremento considerable en el registro de patentes para el periodo 2014-2016, siendo el 88 modelos de utilidad (Z) y 12 patentes de invención (A). Respecto a las líneas de interés tecnológico, las relacionadas con el tratamiento del agua son las más preponderantes. Finalmente, según los hallazgos, se concluye que es necesario fortalecer la colaboración con otras organizaciones e inventores ajenos a la UNI.

The Universidad Nacional de Ingeniería (UNI) through its patents and utility models. Production and network analysis

\section{Abstract}

The study makes visible the collaboration and production networks of patents and utility models of UNI registered in Espacenet until 2019. The Network Analysis (AR) is applied to measure the degree of collaboration of inventors and organizations, as well as the main lines of technological interest. 104 inventors were identified, the collaboration with 2 organizations and 65 lines of technological interest, observing a considerable increase in the registration of patents for the period 2014-2016, being 88 utility models (Z) and 12 invention patents (A). Regarding the lines of technological interest, it is observed that what is related to water treatment is the most important. Finally, according to the findings, collaboration with other organizations and inventors outside the UNI should be strengthened.

\section{Palabras clave}

Análisis de redes Universidad Nacional de Ingeniería (Perú) Patentes

\section{Keywords}

Network analysis Universidad Nacional de Ingeniería (Perú) Patents 
1. El concepto de hélice fue inicialmente desarrollado por Etzkowitz (1993) y Leydesdorff (1995), y se refiere a una mayor colaboración entre el estado, la industria y la academia (Triple Hélice). Posteriormente, se fue ampliando el concepto a cuádruple y quíntuple hélice, ya que incluían la sociedad y el medio ambiente como elementos a considerar.

\section{Introducción}

El rol de la universidad en un mundo globalizado y altamente competitivo es crear talento e investigación de impacto para transformar su entorno, permitiendo la sostenibilidad del desarrollo económico de un país y la consecuente mejora en la calidad de vida de su población (OECD, 2018; CONCYTEC, 2006; CONCYTEC, 2014, UNESCO, 2015, 2018; Rodríguez Ponce, 2009).

Bajo ese contexto, el trabajo académico no debe ser ajeno a las necesidades de su entorno, y debe integrarse a la dinámica del desarrollo tecnológico y productivo. Al respecto, las distintas dinámicas de Hélice ${ }^{1}$ (Academia - Empresa - Estado - Sociedad - Medio ambiente), ha demostrado ser una de las mejores estrategias de trabajo colaborativo entre las instancias mencionadas (OECD, 2018; Martínez de Ita, Piñero y Figueroa Delgado, 2013). Lograr el éxito en esta estrategia colaborativa, depende de la sinergia y sincronía con respecto a intereses en común, bajo un alto espíritu colaborativo y visión conjunta de futuro.

La universidad peruana atraviesa un periodo de cambio acelerado, de adaptación a los requerimientos de competitividad y exigencias tecnológicas de su entorno interno y externo, razón por la cual ha implementado y desarrollado una serie de políticas y estrategias, las que buscan fortalecer su calidad investigativa en ciencia y tecnología, así como sus servicios de enseñanza, lo que le ha permitido ir asumiendo progresivamente un rol cada vez más dinámico en el ecosistema innovador local, así como en constituirse en incubadora de nuevas tecnologías.

A raíz de lo anterior, el Estado Peruano dio la ley número 30200 "Ley universitaria", en cuyo artículo número 4 indica que las universidades del país pueden integrarse a redes interregionales para colaborar con otras organizaciones en líneas de investigación y desarrollo, a fin capturar y aplicar nuevos conocimientos en beneficio del país (Ministerio de Educación, 2014).

Lo indicado por la ley mencionada conlleva a la necesidad de fortalecer las capacidades internas en la universidad peruana, ya que esto es fundamental para integrarse a la dinámica de movilidad del talento y transferencia del conocimiento. Esto exige hacer un diagnóstico interno con respecto a cómo se encuentran las redes colaborativas, los enfoques de las líneas de investigación en ciencia y tecnología, así como las capacidades reales del talento humano.

Respecto a ello, uno de los criterios de calidad considerados por los entes rectores en ciencia y tecnología, así como de calidad universitaria en Perú (CONCYTEC y SUNEDU respectivamente) es la producción de patentes y modelos de utilidad, documentos técnico - jurídicos que reflejan el grado de invención, los intereses tecnológicos y la capacidad del talento nacional, así como su presencia a nivel local e internacional, la cual es aún muy limitada en comparación a otros países de la región (Villarán de la Puente, 2015; SUNEDU, 2018).

La Universidad Nacional de Ingeniería (UNI) fundada el 18 de marzo de 1876, tiene como foco de especialización la formación de ingenieros, siendo reconocida en Perú por su alto nivel de convocatoria y exigencia para cursar estudios en su claustro, siendo a la fecha una de las más importantes universidades nacionales y cantera de catedráticos para otras entidades académicas y empresariales, públicas y privadas.

Por lo anterior, resulta de gran importancia conocer y comprender la dinámica colaborativa al interior de la UNI, así como su relación con su entorno, por lo que el presente estudio busca hacer visible las fortalezas y oportunidades de mejora en el 
trabajo colaborativo y el desarrollo tecnológico reflejado en su producción de patentes y modelos de utilidad.

\section{Análisis de redes}

El análisis de redes es una metodología aplicada en ciencias sociales y estudios de mercado; se caracteriza por usar criterios estadísticos para identificar grados de interacción entre nodos (personas, organizaciones, temas, etc), permitiendo sacar a luz los nodos más importantes en función a las relaciones directas e indirectas, lo cual se refleja en las medidas de centralidad y poder (MCyP) (Valente, 1995; Dima y Vasilache, 2015; Ling, 2009; Liu, 2004; Ma, Jiao y Zhang, 2011; Molina, 2009; Morescalchi et al, 2015; Osca-Lluch, 2010; Stoddart y Siddiqui, 2013; Russell; Madera Jaramillo y Ainsworth, 2009). Para el estudio se consideraron las siguientes medidas de centralidad y poder:

» Número de contactos directos (Degree), corresponde a cuántos contactos directos tiene cada nodo.

" Nodos puente (Between), corresponde a la capacidad de intermediación de un nodo con otros grupos de nodos.

» Nodos de mayor alcance y cercanía (Closeness), se refiere a los nodos que tienen una mayor centralidad en la red.

" Nodos con mayor centralidad y poder (Bonacich), se refiere no solo a los que tienen mayor centralidad, sino que además mayor poder por el grado de sus conexiones.

\section{Materiales y métodos}

Se estudiaron las patentes y modelos de utilidad registradas por la UNI en Espacenet hasta 2019, focalizándose en identificar inventores, organizaciones y líneas de interés tecnológico. Para los efectos, se hizo una revisión exhaustiva de los siguientes campos de Espacenet:

» Número de prioridad: registra la fecha cuando se solicita por primera vez el registro de la invención como patente, por lo que se consideró para delimitar el alcance del estudio.

" Solicitante o aplicante: registra la filiación del inventor y organización que reclama la invención como propia. Aquí se aplicaron los términos de búsqueda “Universidad Nacional de Ingeniería” y “[PE]”para delimitar los registros a analizar a la institución en estudio.

» Clasificación Cooperativa de Patentes (CPC): permite identificar a qué campos tecnológicos aplica cada invención.

Para el análisis y consolidación de resultados se utilizó una base de datos en MS Access, para la aplicación de las MCyP del AR se utilizó el software UCINET y para los gráficos se aplicó el software NETDRAW.

Los resultados se presentan bajo dos enfoques: por ranking de producción y por Análisis de Redes; para ambos se analizaron inventores, organizaciones y líneas de interés tecnológico. Anterior a ello, se presentan los resultados generales como evolución del registro histórico de patentes y modelos de utilidad, presencia de la UNI en países donde se registró su invención y nuevos inventores por año. 


\section{Las patentes de invención y los modelos de utilidad}

En el ámbito de la propiedad intelectual e industrial, ambos documentos son los más importantes, ya que reflejan el estado del arte en distintas disciplinas, cuentan con valor jurídico y documental, así como con una vigencia temporal diferenciada. Si bien protegen y aplican la legislación correspondiente, tienen diferencias, tales como periodo de vigencia, nivel de complejidad de la invención, su reconocimiento o no en todos los países (caso del modelo de utilidad), etc. (OMPI, 2007; Paul y O'Lenick, 2008).

En general, estos documentos están constituidos por seis partes básicas: datos bibliográficos (número del documento, inventor, filiación, fechas, título y resumen), descripción de la invención (detalle de cada parte de la invención), reivindicación de la invención (parte más importante del documento, donde se indica claramente por qué la invención es patentable), mosaicos (dibujos o gráficos de sustento), documento original (archivo en formato pdf) y documentos jurídicos de sustento (Paul y O'Lenick, 2008).

Las patentes se encuentran registradas en bases de datos cuyos campos permiten identificar la información más importante, siendo para Espacenet:

»Título: Nombre de la invención.

» Número de publicación: código unívoco para identificarla, donde se indica el país u organización de solicitud. Ejemplo: PE para Perú, así como el tipo de patente. Por ejemplo: $Z$ o $U$ para modelo de utilidad o $A$ y derivados $\left(A_{1}, A_{2}\right.$, $A_{3}$, etc.) para invención original.

» Número de prioridad: fecha de registro inicial de la invención.

» Fecha de publicación: fecha final, luego de pasar las evaluaciones correspondientes para que la invención sea patentable.

"Solicitante: Nombre de la organización que solicita la patente, así como su ubicación geográfica. Ejemplo: Universidad Nacional de Ingeniería [PE].

» Inventor: Nombres y apellidos de los inventores, así como su nacionalidad. Ejemplo: [PE].

»Clasificaciones: Reflejan las tecnologías a las que aplica la invención, ya que una invención puede aplicar a distintas industrias por transversalidad de la técnica.

El acceso y aplicación de patentes de invención y modelos de utilidad en el desarrollo de nuevas invenciones resulta fundamental, puesto que son fuente de inspiración y punto de inicio para nuevos proyectos; razón por la cual muchas organizaciones cuentan con estrategias para su vigilancia y análisis. Lo anterior conlleva a reformular el rol de la universidad, pasando de ser un ente íntegramente académico, a uno promotor, incubador y generador de nuevas ideas y desarrollos tecnológicos.

\section{Resultados obtenidos}

\section{Registro histórico de patentes y modelos de utilidad}

Se identificaron ciento veinticinco patentes con filiación de la UNI, siendo la más antigua una solicitud de prioridad de patente de invención de 2008. Asimismo, según el campo "Número de publicación" el 100 de estas han sido registradas para ser aplicadas en Perú, de las cuales, 101 (88) corresponden a modelos de utilidad y 24 a patentes de invención. Este es un indicador de que históricamente la estrategia de desarrollo tecnológico de la UNI se ha enfocado en el mercado local y la mejora de soluciones tecnológicas ya implementadas (Modelos de utilidad), dejando en un segundo plano los desarrollos tecnológicos originales. Lo anterior se observa en la Tabla 1. 


\begin{tabular}{|c|c|c|c|}
\hline $\begin{array}{c}\text { Fecha } \\
\text { prioridad }\end{array}$ & $\begin{array}{c}\text { Modelo de } \\
\text { utilidad }\end{array}$ & $\begin{array}{c}\text { Patente de } \\
\text { invención }\end{array}$ & Total \\
\hline 2008 & & 1 & 1 \\
\hline 2009 & 2 & 1 & 3 \\
\hline 2010 & 2 & & 2 \\
\hline 2011 & 1 & 2 & 3 \\
\hline 2012 & 9 & 2 & 11 \\
\hline 2013 & 10 & 1 & 11 \\
\hline 2014 & 26 & 1 & 27 \\
\hline 2015 & 26 & 1 & 27 \\
\hline 2016 & 25 & 2 & 27 \\
\hline 2017 & & 1 & 1 \\
\hline 2018 & & 9 & 9 \\
\hline 2019 & & 3 & 3 \\
\hline Total & 101 & 24 & 125 \\
\hline
\end{tabular}

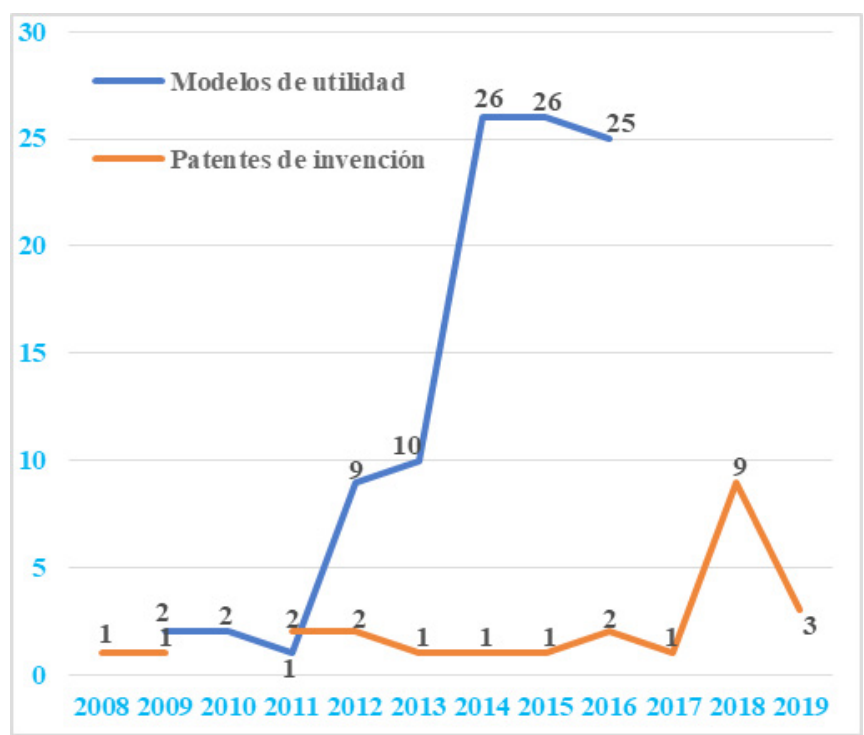

Tabla 1. Patentes por tipo

Según la Tabla 1, se observa un incremento sustancial en el registro de modelos de utilidad en el periodo 2011-2014, manteniéndose estable entre el 2014 y 2016, y disminuye prácticamente desde ese año. En el caso de las patentes de invención, se observa un bajo registro del 2008 al 2017, teniendo un incremento del 2017 al 2018. De lo anterior también se observa que el 65 de patentes solicitadas corresponden al periodo 2014-2016. En general, las patentes de invención han reemplazado a los modelos de utilidad los últimos tres años, ello podría considerarse un indicativo de cambio con respecto a las estrategias de invención y propiedad intelectual en la UNI.

\section{Ranking de inventores y organizaciones}

Según la Tabla 7 (Anexo), al revisar el desempeño histórico respecto al trabajo colaborativo con talento externo, se observa que el 100 de inventores son de nacionalidad peruana, por lo que se advierte un gran reto para la UNI en lo que respecta a trabajo colaborativo externo, si es que quiere competir e integrarse en el ecosistema internacional de la ciencia y tecnología.

En relación con el esfuerzo sostenido de producción de patentes o modelos de utilidad, de acuerdo a la Tabla 2, se observa que la gran mayoría de inventores (86) ha registrado una sola vez una patente, asimismo, la mayoría de equipos de invención ha estado conformado en su mayoría por dos personas. 


\begin{tabular}{rc|c|}
$\begin{array}{r}\text { Registro de } \\
\begin{array}{r}\text { patente o modelo } \\
\text { de utilidad }\end{array}\end{array}$ & \multicolumn{2}{l}{ Inventores } \\
\hline $\begin{array}{r}1 \text { vez } \\
2 \text { veces }\end{array}$ & 99 & 85.6 \\
3 veces & 1 & 8.7 \\
4 veces & 3 & 2.0 \\
5 veces & 1 & 1.0 \\
7 veces & 1 & 1.0 \\
\hline Total & 104 & 100.0
\end{tabular}

Tabla 2. Inventores y equipos de trabajo

\section{Conformación Patentes y \\ de equipos... modelos de \\ utilidad}

\begin{tabular}{lcc}
\hline 1 inventor & 35 & 28 \\
2 inventores & 39 & 31 \\
3 inventores & 21 & 17 \\
4 inventores & 9 & 7 \\
5 inventores & 5 & 4 \\
6 inventores & 4 & 3 \\
7 inventores & 12 & 10 \\
\hline & 125 & 100
\end{tabular}

Sobre la incorporación anual de nuevos inventores (Tabla 3), se observa que durante el periodo 2008-2011 el número de inventores ha sido mínimo, en su totalidad nuevos, incrementándose su número del 2012 al 2016, siendo el 2018 el año donde se ha presentado el mayor número de inventores nuevos, para finalmente descender el 2019. Lo anterior coincide con el cambio de tipo patente publicado según la Tabla 1, lo cual puede deberse a que el perfil del inventor ha cambiado a raíz de que la organización se enfocó en patentes de invención en lugar de modelos de utilidad.

\begin{tabular}{rcccccccccccc} 
& 2008 & 2009 & 2010 & 2011 & 2012 & 2013 & 2014 & 2015 & 2016 & 2017 & 2018 & 2019 \\
\hline $\begin{array}{r}\text { Total de participación } \\
\begin{array}{r}\text { de inventores: } \\
\text { Inventores nuevos: }\end{array}\end{array}$ & 2 & 3 & 3 & 3 & 13 & 13 & 17 & 22 & 34 & 1 & 18 & 5 \\
\hline inventores nuevos & 100 & 100 & 100 & 100 & 77 & 69 & 65 & 55 & 91 & 64 & 94 & 60
\end{tabular}

Tabla 3. Inventores nuevos por año

Respecto al ranking de inventores por publicación de patente, el inventor Walter Héctor Gonzáles Arnao es quien presenta más recurrencia de participación, quien ha publicado 90 patentes ( 26 del total), siendo también quien más participación ha tenido en los grupos de invención. Finalmente, se observa que la mayor participación de inventores se ha dado en el periodo 2014-2016, con 248 participaciones) (Tabla 7 en Anexo).

Respecto a las organizaciones, de acuerdo a la Tabla 4, se ha identificado solo dos patentes en colaboración con otras organizaciones: una con una entidad gubernamental peruana: Autoridad de la Zona Franca de Tacna (ZOTAC) y otra con una universidad privada: Universidad Peruana Cayetano Heredia (UPCH).

\begin{tabular}{|c|c|c|c|c|c|c|c|c|c|c|c|c|c|}
\hline Organización & 2008 & 2009 & 2010 & 2011 & 2012 & 2013 & \multicolumn{2}{|c|}{2014} & 2015 & 2016 & 2017 & 2018 & 2019 \\
\hline \multicolumn{14}{|l|}{ UPCH } \\
\hline UNI & 1 & 3 & 2 & 3 & 11 & 11 & \multirow{2}{*}{1} & 26 & 27 & 27 & 1 & 9 & 3 \\
\hline ZOTAC & & & & & & & & & & & & & \\
\hline
\end{tabular}

Tabla 4 - Colaboración con otras organizaciones 


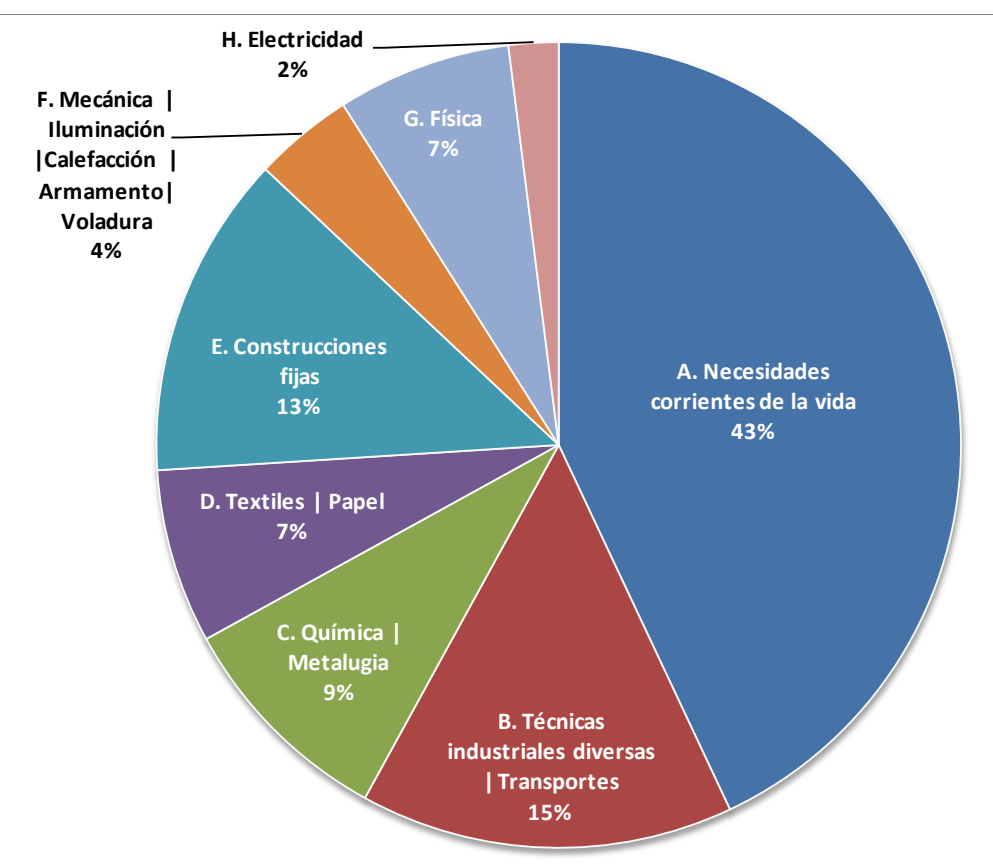

Gráfico 1 - Líneas de interés tecnológico

En función a los resultados de la Tabla 4, al igual que los inventores, se observa una gran oportunidad con respecto a buscar una mayor colaboración con inventores y organizaciones nacionales y extranjeras, tanto académicas como del sector industrial; asimismo, a raíz de este resultado, no sería necesario realizar un AR con respecto a organizaciones.

\section{Rankings de líneas de interés tecnológico}

Según el Gráfico 1, 43 de las invenciones aplican a necesidades humanas (Sección A) y 15 a técnicas industriales diversas (Sección B). En el primer caso, la mayor cantidad corresponde a sillas, sofás y camas $(\mathrm{A} 47 \mathrm{C})$, tiovivos; columpios; caballos basculantes; toboganes; montaña rusa; seguida de distracciones publicas análogas ( $\mathrm{A} 63 \mathrm{G})$ y mesas; escritorios; mobiliario de oficina; muebles con cajones; cajones; partes constitutivas generales de los muebles (A47B). (Tabla 8 en Anexo).

\section{Análisis de redes de inventores}

La aplicación de las MCyP en este estudio hace visible a los inventores, organizaciones y líneas de interés tecnológico más importantes, en base a su grado de alcance y relación en la red colaborativa, lo que permite validar los rankings obtenidos en función al número de ocurrencias reflejado en la Tabla 7 de la sección de Anexo. Al respecto, se consideró a la MCyP la de Bonacich como principal criterio para el ordenamiento de los resultados, tal como lo muestra la Tabla 5 en Anexo.

Walter Héctor Gonzáles Arnao tiene los mayores valores en todas las MCyP, seguido de Miguel Ángel Vidal Valladolid. En el primer caso, existe coincidencia con los resultados de la Tabla 7. Con respecto al segundo inventor, este se encuentra en el puesto 10 en esta Tabla, ello a raíz de no haber tenido un mayor número de publicaciones; sin embargo, su ubicación en el segundo puesto se debe a que ha tenido un mayor trabajo colaborativo (reflejado en la MCyP). 


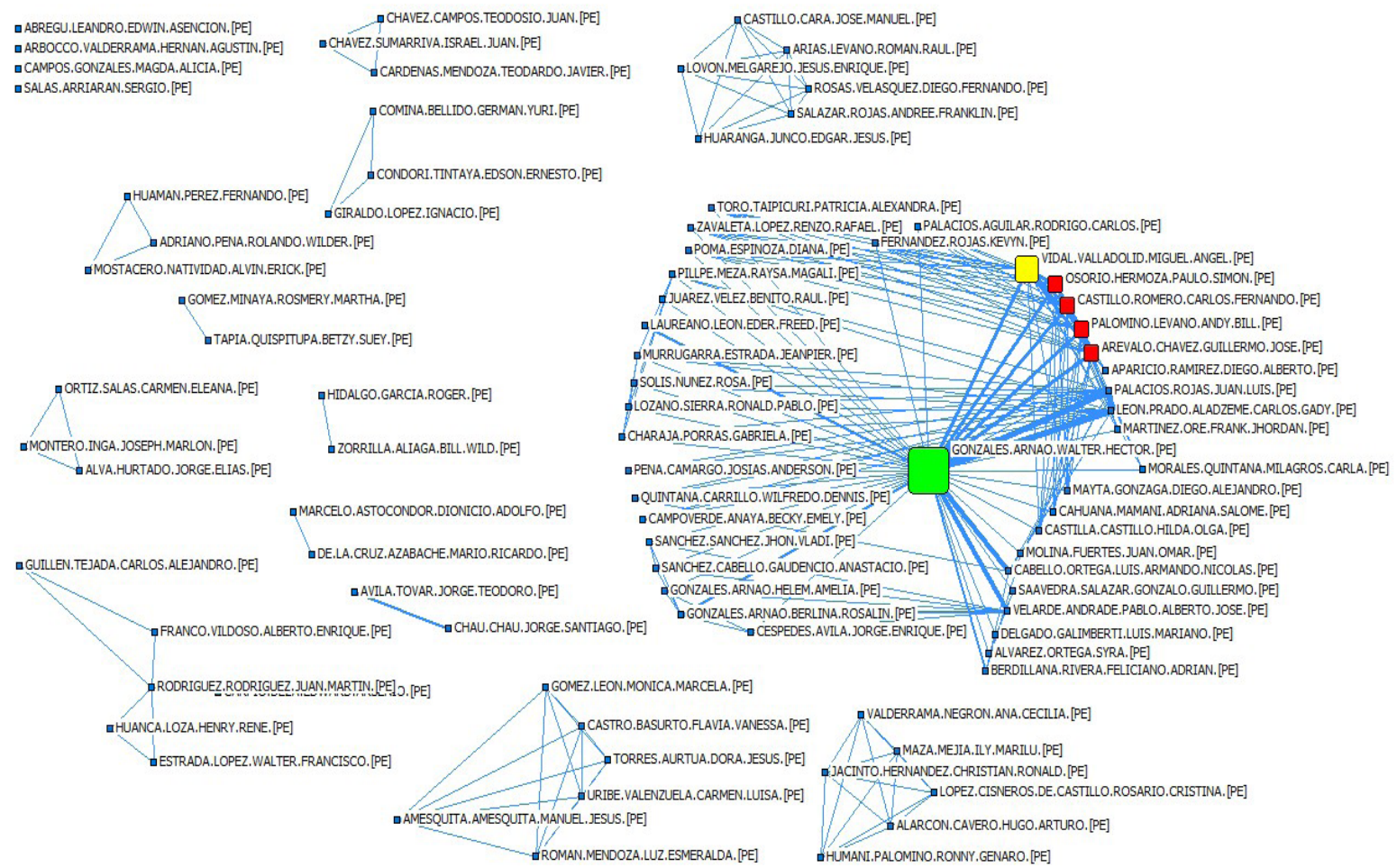

Gráfico 2. Red de inventores

Por otra parte, según el Gráfico 2, se observa un grupo colaborativo altamente relacionado, comprendido por los investigadores Gonzáles Arnao, Arévalo Chávez, Osorio Hermoza, Castillo Romero, Palomino Lévano y Vidal Valladolid. Todos aglutinados por el primer inventor. Esto también se refleja en la Tabla 5 y en parte en la Tabla 7. Con lo anterior se hace visible el principal grupo colaborativo de la UNI, que a su vez tuvo su mayor dinamismo en el periodo 2014-2016.

\section{Análisis de redes de líneas de interés tecnológico}

Se visibiliza que la mayor línea de interés tecnológico de la UNI corresponde a invenciones sobre tratamiento del agua ( $\mathrm{Co} 2 \mathrm{~F})$, así como la tecnología relacionada, tales como procedimientos o aparatos físico o químicos (Bo1D), procedimientos o aparatos para esterilización $(\mathrm{A} 61 \mathrm{~L})$, polisacáridos $(\mathrm{Co8B})$ y pasteurización $(\mathrm{C} 12 \mathrm{H})$. Lo anterior se aprecia en la Tabla 6 y en el Gráfico 3. 


\begin{tabular}{|c|c|c|c|c|c|}
\hline & CLASIFICACIÓN & D & $\mathrm{BO}$ & C & B \\
\hline 1 & $\mathrm{Co} 2 \mathrm{~F}$ - tratamiento del agua, agua residual, de alcantarilla o fangos & 4 & 642 & 258 & 9 \\
\hline 2 & Bo1D - procedimientos o aparatos físicos o químicos en general - separación & 2 & 403 & 260 & 4 \\
\hline 3 & $\begin{array}{l}\text { A61L - procedimientos o aparatos para esterilizar materiales u objetos en } \\
\text { general }\end{array}$ & 1 & 309 & 262 & 0 \\
\hline 4 & Co8B - polisacáridos; sus derivados & 1 & 309 & 262 & o \\
\hline 5 & $\begin{array}{l}\text { Ho1M - Procedimientos o medios, p. ej. baterías, para la conversión directa de } \\
\text { la energía química en energía eléctrica }\end{array}$ & 1 & 309 & 262 & 0 \\
\hline 6 & $\begin{array}{l}\text { C12H - pasteurización, esterilización, conservación, purificación, clarificación o } \\
\text { envejecimiento de bebidas alcohólicas; métodos para alterar el contenido de } \\
\text { alcohol de las soluciones fermentadas o de las bebidas alcohólicas }\end{array}$ & 1 & 194 & 264 & 0 \\
\hline 7 & A47C - sillas; sofás; camas & 2 & 5 & 266 & 1 \\
\hline 8 & $\mathrm{~A} 47 \mathrm{~L}$ - lavado o limpieza domestica; aspiradores en general & 2 & 5 & 266 & 1 \\
\hline 9 & B62D - vehículos de motor; remolques & 2 & 5 & 266 & 1 \\
\hline 10 & Do3D - tejidos; métodos de tejido; máquinas para tejer & 2 & 5 & 266 & 1 \\
\hline 11 & $\begin{array}{l}\text { A45F - material de viaje o de camping; sacos o paquetes llevados sobre el } \\
\text { cuerpo }\end{array}$ & 1 & 4 & 267 & 0 \\
\hline 12 & A46B - cepillos o pinceles & 1 & 4 & 267 & o \\
\hline 13 & $\begin{array}{l}\text { A63G - tiovivos; columpios; caballos basculantes; toboganes; montaña rusa; } \\
\text { distracciones publicas análogas }\end{array}$ & 1 & 4 & 267 & 0 \\
\hline 14 & A63H - juguetes, p. Ej. Trompos, muñecos, aros, juegos de construcción & 1 & 4 & 267 & 0 \\
\hline 15 & B63 - buques u otras embarcaciones flotantes, propulsión o gobierno marino & 1 & 4 & 267 & 0 \\
\hline 16 & $\begin{array}{l}\text { Eo1H - limpieza de calles; limpieza de vías férreas; limpieza de playas; limpieza } \\
\text { de terrenos; dispersión de niebla en general }\end{array}$ & 1 & 4 & 267 & 0 \\
\hline 17 & $\begin{array}{l}\text { Eo4G - andamiajes; moldes; encofrados; instrumentos para la construcción o } \\
\text { cualquier otro material }\end{array}$ & 1 & 4 & 267 & 0 \\
\hline 18 & $\begin{array}{l}\text { GogB - aparatos de cifrar o descifrar para la criptografía u otros fines que } \\
\text { implican la necesidad del secreto }\end{array}$ & 1 & 4 & 267 & 0 \\
\hline 19 & A $47 \mathrm{G}$ - utensilios de uso doméstico o de mesa & 1 & 2 & 269 & 0 \\
\hline 20 & A61B - diagnostico; cirugía; identificación & 1 & 2 & 269 & o \\
\hline
\end{tabular}

Tabla 6. Top 20 líneas de interés tecnológico 


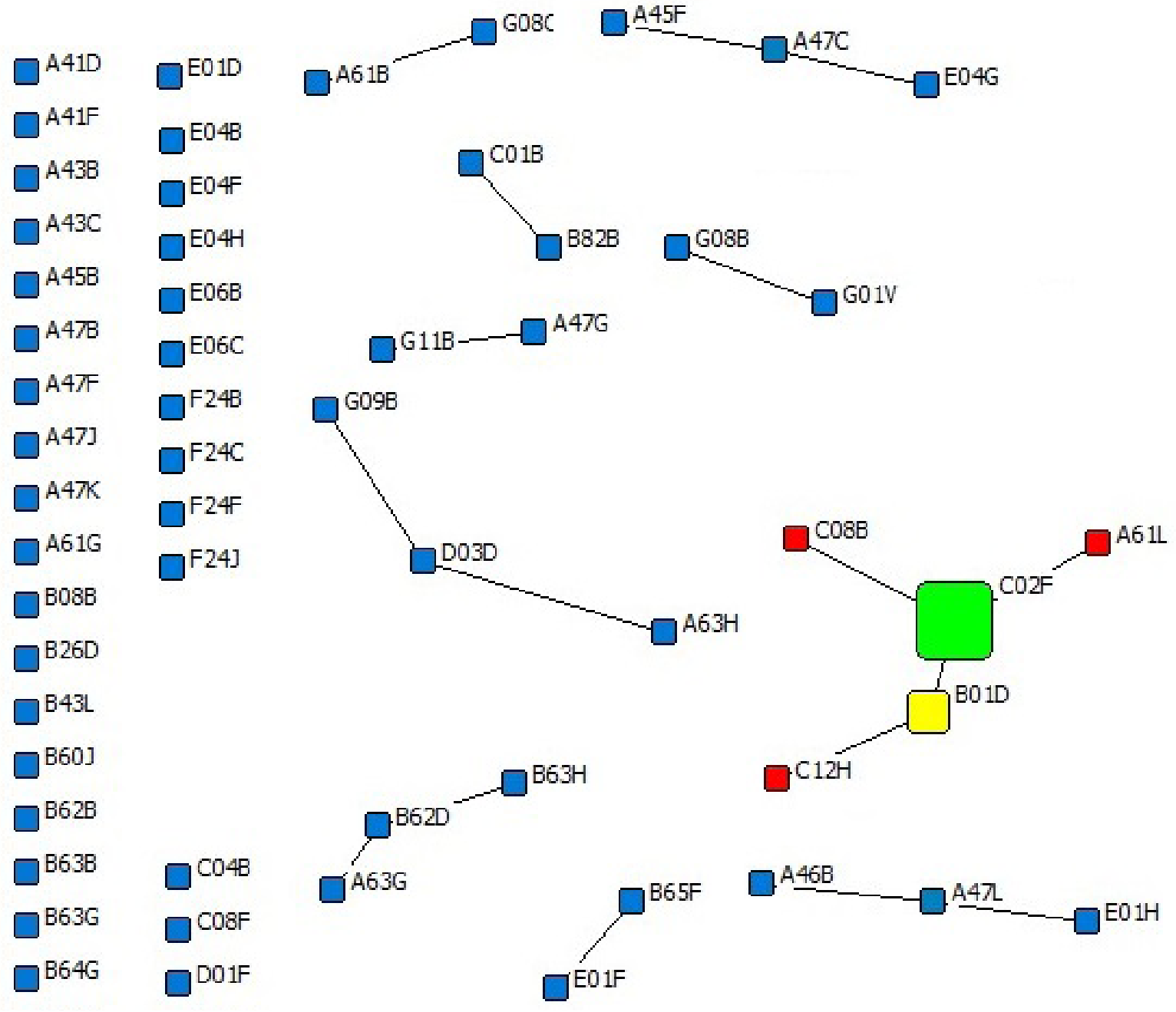

Gráfico 3. Red de líneas de interés temático

De acuerdo a los resultados de las MCyP, aplicados a líneas de interés tecnológico, la UNI tiene un interés mayor sobre tratamiento de aguas y temas derivados, y no sobre sillas, sofás o camas; resultado obtenido mediante el conteo de ocurrencias (Tabla 8-Anexo). Lo anterior reafirma la importancia del Análisis de Redes en validar resultados preliminares al momento de identificar lo realmente importante en función del enfoque colaborativo.

\section{Conclusiones}

"Sobre los resultados, destaca la necesidad de fortalecer mucho más el trabajo colaborativo de la UNI con otras entidades, tanto nacionales como externas, teniendo en consideración los altos niveles de competitividad y alta movilidad del talento que existe en la región. 
"Sobre el registro de solicitudes de patentes (según el campo fecha de prioridad), se observa que luego del periodo de mayor dinámica (2014-2016), el registro de patentes ha descendido considerablemente (sólo 3 patentes en 2019). Lo anterior lleva a una reflexión sobre el enfoque y la sostenibilidad del esfuerzo de invención al interior de la organización objeto de estudio.

"El 59 de equipos de trabajo se conforma de uno o dos inventores, lo que refleja una gran oportunidad para mejorar el nivel colaborativo interno, teniendo además en consideración que el 85.6 de inventores ha registrado solo una participación en la solicitud de patentes de invención y modelos de utilidad.

» Respecto a la metodología, aplicar el AR permite realizar un estudio más profundo con respecto a los grados de influencia y alcance entre los integrantes de una red. Lo anterior, es fundamental para tomar decisiones correctas con respecto a donde focalizar el trabajo colaborativo.

\section{Recomendaciones}

" Siendo este el primer estudio que hace visible la dinámica colaborativa de la UNI, se sugiere continuar con su actualización periódica, a fin de contar con información permanente de la evolución de sus redes colaborativas; ello permitirá orientar las decisiones estratégicas con respecto a nuevos desarrollos tecnológicos e integración a ecosistemas de innovación.

» La UNI por su importancia en la formación de talento y generación de nuevos conocimientos en ingeniería para el Perú, no puede ser ajena a integrarse a la dinámica colaborativa con otras organizaciones e investigadores. Razón por la cual debe establecer planes de colaboración y producción de patentes de manera sostenible, a fin de ir construyendo un Know-How propio, competitivo y de impacto para el desarrollo del país. 


\section{Q Anexo}

Tabla 5. Inventores UNI bajo MCyP

\begin{tabular}{|c|c|c|c|c|c|}
\hline & INVENTORES & D & $\mathrm{BO}$ & $\mathrm{C}$ & B \\
\hline 1 & GONZALES.ARNAO.WALTER.HECTOR.[PE] & 169 & 23423 & 229 & 596 \\
\hline 2 & VIDAL.VALLADOLID.MIGUEL.ANGEL.[PE] & 66 & 16377 & 254 & 8 \\
\hline 3 & AREVALO.CHAVEZ.GUILLERMO.JOSE.[PE] & 66 & 15919 & 253 & 10 \\
\hline 4 & CASTILLO.ROMERO.CARLOS.FERNANDO.[PE] & 66 & 15919 & 253 & 10 \\
\hline 5 & OSORIO.HERMOZA.PAULO.SIMON.[PE] & 66 & 15919 & 253 & 10 \\
\hline 6 & PALOMINO.LEVANO.ANDY.BILL.[PE] & 66 & 15919 & 253 & 10 \\
\hline 7 & LEON.PRADO.ALADZEME.CARLOS.GADY.[PE] & 46 & 9485 & 257 & 13 \\
\hline 8 & PALACIOS.ROJAS.JUAN.LUIS.[PE] & 46 & 9485 & 257 & 13 \\
\hline 9 & CABELLO.ORTEGA.LUIS.ARMANDO.NICOLAS.[PE] & 21 & 5910 & 264 & 1 \\
\hline 10 & VELARDE.ANDRADE.PABLO.ALBERTO.JOSE.[PE] & 26 & 5627 & 263 & 1 \\
\hline 11 & BERDILLANA.RIVERA.FELICIANO.ADRIAN.[PE] & 9 & 2423 & 266 & $\mathbf{o}$ \\
\hline 12 & LAUREANO.LEON.EDER.FREED.[PE] & 6 & 2276 & 268 & $\mathbf{o}$ \\
\hline 13 & GONZALES.ARNAO.BERLINA.ROSALIN.[PE] & 16 & 2064 & 263 & 1 \\
\hline 14 & MARTINEZ.ORE.FRANK.JHORDAN.[PE] & 7 & 1943 & 263 & $\mathbf{o}$ \\
\hline 15 & APARICIO.RAMIREZ.DIEGO.ALBERTO.[PE] & 6 & 1677 & 263 & $\mathbf{o}$ \\
\hline 16 & CASTILLA.CASTILLO.HILDA.OLGA.[PE] & 6 & 1677 & 263 & o \\
\hline 17 & FERNANDEZ.ROJAS.KEVYN.[PE] & 6 & 1677 & 263 & o \\
\hline 18 & MAYTA.GONZAGA.DIEGO.ALEJANDRO.[PE] & 6 & 1677 & 263 & o \\
\hline 19 & MORALES.QUINTANA.MILAGROS.CARLA.[PE] & 6 & 1677 & 263 & $\mathbf{o}$ \\
\hline 20 & PALACIOS.AGUILAR.RODRIGO.CARLOS.[PE] & 6 & 1677 & 263 & o \\
\hline 21 & POMA.ESPINOZA.DIANA.[PE] & 6 & 1677 & 263 & $\mathbf{o}$ \\
\hline 22 & TORO.TAIPICURI.PATRICIA.ALEXANDRA.[PE] & 6 & 1677 & 263 & o \\
\hline 23 & ZAVALETA.LOPEZ.RENZO.RAFAEL.[PE] & 6 & 1677 & 263 & $\mathbf{o}$ \\
\hline 24 & SANCHEZ.CABELLO.GAUDENCIO.ANASTACIO.[PE] & 12 & 1597 & 265 & $\mathbf{o}$ \\
\hline 25 & SANCHEZ.SANCHEZ.JHON.VLADI.[PE] & 12 & 1597 & 265 & $\mathbf{o}$ \\
\hline 26 & MOLINA.FUERTES.JUAN.OMAR.[PE] & 7 & 1527 & 265 & 1 \\
\hline 27 & CAHUANA.MAMANI.ADRIANA.SALOME.[PE] & 5 & 1412 & 264 & $\mathbf{o}$ \\
\hline 28 & ALVAREZ.ORTEGA.SYRA.[PE] & 6 & 815 & 266 & $\mathbf{o}$ \\
\hline 29 & DELGADO.GALIMBERTI.LUIS.MARIANO.[PE] & 6 & 815 & 266 & o \\
\hline 30 & SAAVEDRA.SALAZAR.GONZALO.GUILLERMO.[PE] & 6 & 815 & 266 & $\mathbf{o}$ \\
\hline 31 & QUINTANA.CARRILLO.WILFREDO.DENNIS.[PE] & 4 & 784 & 265 & $\mathbf{o}$ \\
\hline 32 & CHARAJA.PORRAS.GABRIELA.[PE] & 6 & 726 & 263 & $\mathbf{o}$ \\
\hline 33 & LOZANO.SIERRA.RONALD.PABLO.[PE] & 6 & 726 & 263 & $\mathbf{o}$ \\
\hline 34 & MURRUGARRA.ESTRADA.JEANPIER.[PE] & 6 & 726 & 263 & $\mathbf{o}$ \\
\hline 35 & PILLPE.MEZA.RAYSA.MAGALI.[PE] & 6 & 726 & 263 & $\mathbf{o}$ \\
\hline 36 & CAMPOVERDE.ANAYA.BECKY.EMELY.[PE] & 3 & 688 & 266 & o \\
\hline
\end{tabular}




\begin{tabular}{|c|c|}
\hline 37 & PENA.CAMARGO.JOSIAS.ANDERSON.[PE] \\
\hline 38 & SOLIS.NUNEZ.ROSA.[PE] \\
\hline 39 & CESPEDES.AVILA.JORGE.ENRIQUE.[PE] \\
\hline 40 & GONZALES.ARNAO.HELEM.AMELIA.[PE] \\
\hline 41 & JUAREZ.VELEZ.BENITO.RAUL.[PE] \\
\hline 42 & BAENA.MONCADA.ANGELICA.MARIA.[PE] \\
\hline 43 & LA.ROSA.TORO.GOMEZ.ADOLFO.[PE] \\
\hline 44 & RODRIGUEZ.RODRIGUEZ.JUAN.MARTIN.[PE] \\
\hline 45 & CARDENAS.RIOJAS.ANDY.ALFREDO.[PE] \\
\hline 46 & CORNEJO.HERRERA.ANTHONY.FELIX.[PE] \\
\hline 47 & MUEDAS.TAIPE.GOLFER.[PE] \\
\hline 48 & TABOADA.SOTOMAYOR.MARIA.DEL.PILAR.[PE] \\
\hline 49 & ALARCON.CAVERO.HUGO.ARTURO.[PE] \\
\hline 50 & AMESQUITA.AMESQUITA.MANUEL.JESUS.[PE] \\
\hline 51 & ARIAS.LEVANO.ROMAN.RAUL.[PE] \\
\hline 52 & CASTILLO.CARA.JOSE.MANUEL.[PE] \\
\hline 53 & CASTRO.BASURTO.FLAVIA.VANESSA.[PE] \\
\hline 54 & GOMEZ.LEON.MONICA.MARCELA.[PE] \\
\hline 55 & HUARANGA.JUNCO.EDGAR.JESUS.[PE] \\
\hline 56 & HUMANI.PALOMINO.RONNY.GENARO.[PE] \\
\hline 8 & JACINTO.HERNANDEZ.CHRISTIAN.RONALD.[PE] \\
\hline 58 & LOPEZ.CISNEROS.DE.CASTILLO.ROSARIO.CRISTINA.[PE] \\
\hline 59 & LOVON.MELGAREJO.JESUS.ENRIQUE.[PE] \\
\hline 60 & MAZA.MEJIA.ILY.MARILU.[PE] \\
\hline 61 & ROMAN.MENDOZA.LUZ.ESMERALDA.[PE] \\
\hline 62 & ROSAS.VELASQUEZ.DIEGO.FERNANDO.[PE] \\
\hline 63 & SALAZAR.ROJAS.ANDREE.FRANKLIN.[PE] \\
\hline 64 & TORRES.AURTUA.DORA.JESUS.[PE] \\
\hline 65 & URIBE.VALENZUELA.CARMEN.LUISA.[PE] \\
\hline 66 & VALDERRAMA.NEGRON.ANA.CECILIA.[PE] \\
\hline 67 & ADRIANO.PENA.ROLANDO.WILDER.[PE] \\
\hline 68 & HUAMAN.PEREZ.FERNANDO.[PE] \\
\hline 69 & MOSTACERO.NATIVIDAD.ALVIN.ERICK.[PE] \\
\hline 70 & AVILA.TOVAR.JORGE.TEODORO.[PE] \\
\hline 71 & CHAU.CHAU.JORGE.SANTIAGO.[PE] \\
\hline 72 & AGREDA.TURRIATE.ISAURO.CARLOS.[PE] \\
\hline 73 & GALLARDO.COZ.ERIKA.RAQQUEL.[PE] \\
\hline 74 & TEVES.ROJAS.AUGUSTO.[PE] \\
\hline 75 & URQUIZO.ARAUJO.ADAN.MAK.BRAYAM.[PE] \\
\hline 76 & CALDERON.ZAVALETA.SANDY.LUZ.[PE] \\
\hline 77 & QUIROZ.AGUINAGA.ULISES.[PE] \\
\hline 78 & CARPIO.DEZA.EDWARD.ARSENIO.[PE] \\
\hline 79 & ESTRADA.LOPEZ.WALTER.FRANCISCO.[PE] \\
\hline
\end{tabular}

\begin{tabular}{|c|c|c|c|}
\hline 3 & 688 & 266 & o \\
\hline 3 & 688 & 266 & o \\
\hline 4 & 515 & 265 & o \\
\hline 4 & 515 & 265 & 0 \\
\hline 1 & 379 & 268 & 0 \\
\hline 9 & 10 & 295 & 5 \\
\hline 9 & 10 & 295 & 5 \\
\hline 6 & 6 & 299 & 8 \\
\hline 5 & 6 & 297 & 0 \\
\hline 5 & 6 & 297 & o \\
\hline 5 & 6 & 297 & 0 \\
\hline 5 & 6 & 297 & 0 \\
\hline 5 & 5 & 299 & 0 \\
\hline 5 & 5 & 299 & o \\
\hline 5 & 5 & 299 & o \\
\hline 5 & 5 & 299 & 0 \\
\hline 5 & 5 & 299 & 0 \\
\hline 5 & 5 & 299 & 0 \\
\hline 5 & 5 & 299 & o \\
\hline 5 & 5 & 299 & o \\
\hline 5 & 5 & 299 & 0 \\
\hline 5 & 5 & 299 & o \\
\hline 5 & 5 & 299 & 0 \\
\hline 5 & 5 & 299 & 0 \\
\hline 5 & 5 & 299 & 0 \\
\hline 5 & 5 & 299 & 0 \\
\hline 5 & 5 & 299 & 0 \\
\hline 5 & 5 & 299 & 0 \\
\hline 5 & 5 & 299 & o \\
\hline 5 & 5 & 299 & o \\
\hline 4 & 4 & 305 & o \\
\hline 4 & 4 & 305 & o \\
\hline 4 & 4 & 305 & o \\
\hline 4 & 4 & 307 & o \\
\hline 4 & 4 & 307 & o \\
\hline 3 & 3 & 303 & o \\
\hline 3 & 3 & 303 & o \\
\hline 3 & 3 & 303 & o \\
\hline 3 & 3 & 303 & o \\
\hline 2 & 2 & 300 & 0 \\
\hline 2 & 2 & 300 & 0 \\
\hline 2 & 2 & 303 & 0 \\
\hline 2 & 2 & 302 & o \\
\hline
\end{tabular}




\begin{tabular}{|c|c|c|c|c|c|}
\hline 80 & FRANCO.VILDOSO.ALBERTO.ENRIQUE.[PE] & 2 & 2 & 302 & o \\
\hline 81 & GUILLEN.TEJADA.CARLOS.ALEJANDRO.[PE] & 2 & 2 & 302 & o \\
\hline 82 & HUANCA.LOZA.HENRY.RENE.[PE] & 2 & 2 & 302 & o \\
\hline 83 & ALVA.HURTADO.JORGE.ELIAS.[PE] & 2 & 2 & 305 & o \\
\hline 84 & CARDENAS.MENDOZA.TEODARDO.JAVIER.[PE] & 2 & 2 & 305 & o \\
\hline 85 & CHAVEZ.CAMPOS.TEODOSIO.JUAN.[PE] & 2 & 2 & 305 & o \\
\hline 86 & CHAVEZ.SUMARRIVA.ISRAEL.JUAN.[PE] & 2 & 2 & 305 & o \\
\hline 87 & COMINA.BELLIDO.GERMAN.YURI.[PE] & 2 & 2 & 305 & o \\
\hline 88 & CONDORI.TINTAYA.EDSON.ERNESTO.[PE] & 2 & 2 & 305 & o \\
\hline 89 & GIRALDO.LOPEZ.IGNACIO.[PE] & 2 & 2 & 305 & o \\
\hline 90 & MONTERO.INGA.JOSEPH.MARLON.[PE] & 2 & 2 & 305 & o \\
\hline 91 & ORTIZ.SALAS.CARMEN.ELEANA.[PE] & 2 & 2 & 305 & o \\
\hline 92 & DE.LA.CRUZ.AZABACHE.MARIO.RICARDO.[PE] & 1 & 1 & 307 & o \\
\hline 93 & FERNANDEZ.CORI.REY.ANTONIO.[PE] & 1 & 1 & 307 & o \\
\hline 94 & GOMEZ.MINAYA.ROSMERY.MARTHA.[PE] & 1 & 1 & 307 & o \\
\hline 95 & HIDALGO.GARCIA.ROGER.[PE] & 1 & 1 & 307 & o \\
\hline 96 & MARCELO.ASTOCONDOR.DIONICIO.ADOLFO.[PE] & 1 & 1 & 307 & o \\
\hline 97 & MORALES.GOMERO.JUAN.CARLOS.[PE] & 1 & 1 & 307 & o \\
\hline 98 & TAPIA.QUISPITUPA.BETZY.SUEY.[PE] & 1 & 1 & 307 & o \\
\hline 99 & ZORRILLA.ALIAGA.BILL.WILD.[PE] & 1 & 1 & 307 & o \\
\hline 100 & ABREGU.LEANDRO.EDWIN.ASENCION.[PE] & o & $\mathbf{o}$ & 309 & o \\
\hline 101 & ARBOCCO.VALDERRAMA.HERNAN.AGUSTIN.[PE] & o & o & 309 & o \\
\hline 102 & CAMPOS.GONZALES.MAGDA.ALICIA.[PE] & o & o & 309 & o \\
\hline 103 & RIOS.JULCAPOMA.MILTON.[PE] & o & o & 309 & o \\
\hline 104 & SALAS.ARRIARAN.SERGIO.[PE] & o & o & 309 & o \\
\hline
\end{tabular}


Tabla 7. Patentes publicadas por inventor

Nuevo inventor

Inventor que ya ha publicado

\begin{tabular}{|c|c|c|c|c|c|c|c|c|c|c|c|c|c|c|c|c|}
\hline & Inventores & 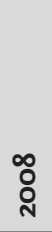 & $\begin{array}{l}\text { ஜ } \\
\text { ঠ্ }\end{array}$ & 윰 & 고ํ & กี้ & กิ & ষ্ণ & กิ กิ & 유 & คิ & م્స & बें & 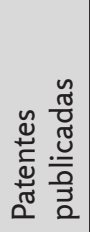 & $b^{\circ}$ & 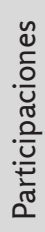 \\
\hline 1 & Gonzáles Arnao Walter Hector [PE] & & 1 & & & 8 & 10 & 22 & 24 & 24 & 1 & & & 90 & 26 & 7 \\
\hline 2 & Palacios Rojas Juan Luis [PE] & & & & & 1 & 3 & 7 & 6 & & & & & 17 & 5 & 4 \\
\hline 3 & León Prado Ladzeme Carlos Gady [PE] & & & & & 1 & 3 & 7 & 6 & & & & & 17 & 5 & 4 \\
\hline 4 & Velarde Andrade Pablo Alberto José [PE] & & 1 & & & 2 & 2 & 5 & 4 & & & & & 14 & 4 & 5 \\
\hline 5 & Cabello Ortega Luis Armando Nicolas [PE] & & & & & & 3 & 9 & 1 & & & & & 13 & 4 & 3 \\
\hline 6 & Arevalo Chávez Guillermo José [PE] & & & & & & & & & 11 & & & & 11 & 3 & 1 \\
\hline 7 & Castillo Romero Carlos Fernando [PE] & & & & & & & & & 11 & & & & 11 & 3 & 1 \\
\hline 8 & Osorio Hermoza Paulo Simón [PE] & & & & & & & & & 11 & & & & 11 & 3 & 1 \\
\hline 9 & Palomino Lévano Andy Bill [PE] & & & & & & & & & 11 & & & & 11 & 3 & 1 \\
\hline 10 & Vidal Valladolid Miguel Angel [PE] & & & & & & & & & 11 & & & & 11 & 3 & 1 \\
\hline 11 & Berdillana Rivera Feliciano Adrián [PE] & & & & & & 1 & 2 & 2 & 1 & & & & 6 & 2 & 4 \\
\hline 12 & Laureano León Eder Freed [PE] & & & & & & & & 5 & 1 & & & & 6 & 2 & 2 \\
\hline 13 & De la Cruz Azabache Mario Ricardo [PE] & 1 & & & & & & & & & & 4 & & 5 & 1 & 2 \\
\hline 14 & Avila Tovar Jorge Teodoro [PE] & & & & & & & 4 & & & & & & 4 & 1 & 1 \\
\hline 15 & Chau Chau Jorge Santiago [PE] & & & & & & & 4 & & & & & & 4 & 1 & 1 \\
\hline 16 & Gonzáles Arnao Berlina Rosalin [PE] & & & & & & & 2 & 2 & & & & & 4 & 1 & 2 \\
\hline 17 & Rodriguez Rodríguez Juan Martin [PE] & & & & 2 & 2 & & & & & & & & 4 & 1 & 2 \\
\hline 18 & Baena Moncada Angelica Maria [PE] & & & & & & & & & & & 1 & 2 & 3 & 1 & 2 \\
\hline 19 & La Rosa Toro Gómez Adolfo [PE] & & & & & & & & & & & 1 & 2 & 3 & 1 & 2 \\
\hline 20 & Molina Fuertes Juan Omar [PE] & & & & & & & & 3 & & & & & 3 & 1 & 1 \\
\hline 21 & Sanchez Cabello Gaudencio Anastacio [PE] & & & & & & & 1 & 2 & & & & & 3 & 1 & 2 \\
\hline 22 & Sanchez Sanchez Jhon Vladi [PE] & & & & & & & 1 & 2 & & & & & 3 & 1 & 2 \\
\hline 23 & Adriano Peña Rolando Wilder [PE] & & & 2 & & & & & & & & & & 2 & 1 & 1 \\
\hline 24 & Álvarez Ortega Syra [PE] & & & & & & & & 2 & & & & & 2 & 1 & 1 \\
\hline 25 & Arbocco Valderrama Hernán Agustín [PE] & & & & & & 1 & & 1 & & & & & 2 & 1 & 2 \\
\hline 26 & Carpio Deza Edward Arsenio [PE] & & & & 2 & & & & & & & & & 2 & 1 & 1 \\
\hline 27 & Delgado Galimberti Luis Mariano [PE] & & & & & & & & 2 & & & & & 2 & 1 & 1 \\
\hline 28 & Huaman Perez Fernando [PE] & & & 2 & & & & & & & & & & 2 & 1 & 1 \\
\hline 29 & Mostacero Natividad Alvin Erick [PE] & & & 2 & & & & & & & & & & 2 & 1 & 1 \\
\hline 30 & Saavedra Salazar Gonzalo Guillermo [PE] & & & & & & & & 2 & & & & & 2 & 1 & 1 \\
\hline 31 & Salas Arriaran Sergio [PE] & & 2 & & & & & & & & & & & 2 & 1 & 1 \\
\hline 32 & Abregu Leandro Edwin Asencion [PE] & & & & 1 & & & & & & & & & 1 & o & 1 \\
\hline 33 & Agreda Turriate Isauro Carlos [PE] & & & & & & & & & & & 1 & & 1 & 0 & 1 \\
\hline 34 & Alarcon Cavero Hugo Arturo [PE] & & & & & & & & & 1 & & & & 1 & 0 & 1 \\
\hline 35 & Alva Hurtado Jorge Elías [PE] & & & & & & & & & & & 1 & & 1 & o & 1 \\
\hline
\end{tabular}




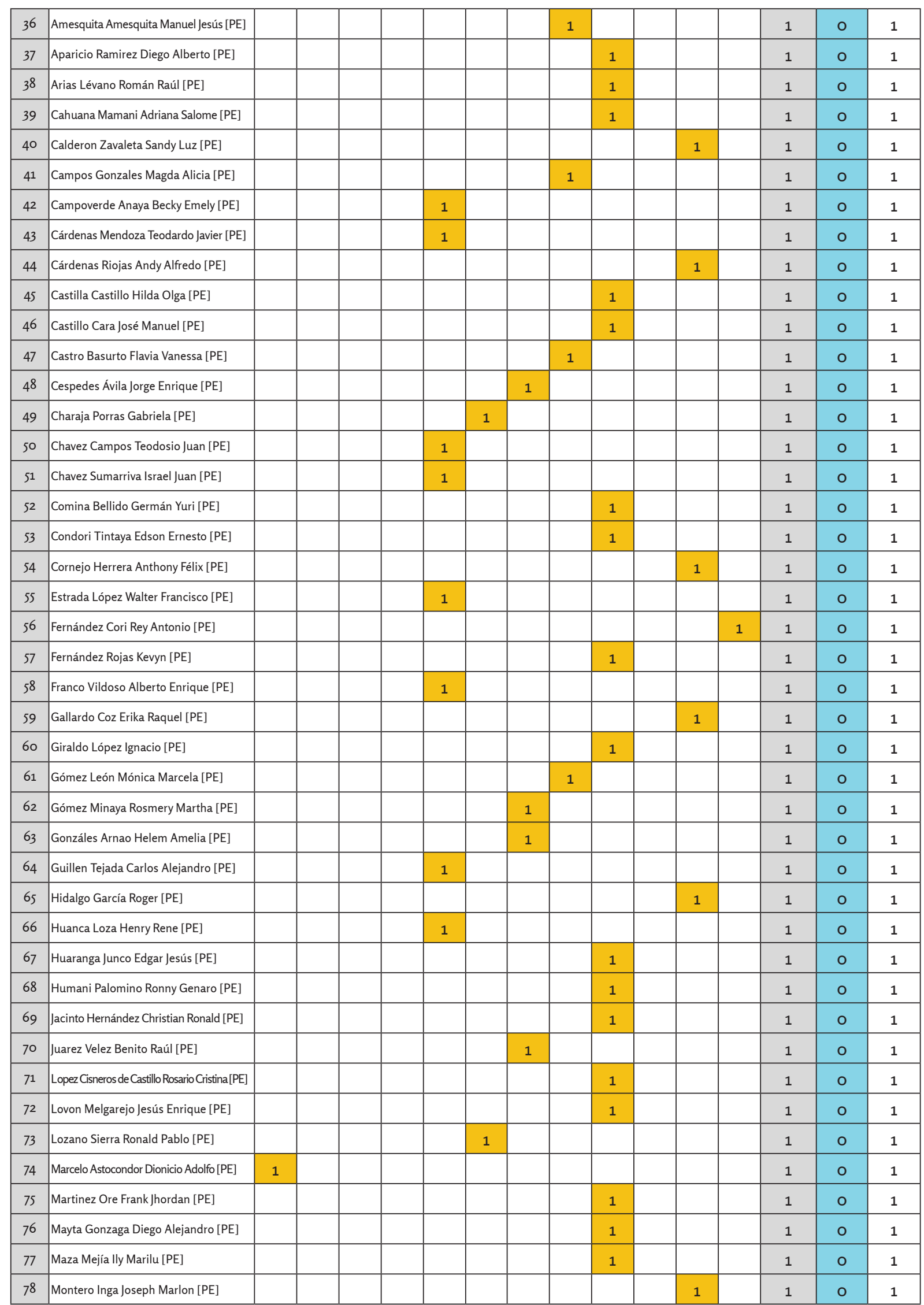




\begin{tabular}{|c|c|c|c|c|c|c|c|c|c|c|c|c|c|c|c|c|}
\hline 79 & Morales Gomero Juan Carlos [PE] & & & & & & & & & & & & 1 & 1 & $\mathrm{O}$ & 1 \\
\hline 80 & Morales Quintana Milagros Carla [PE] & & & & & & & & & 1 & & & & 1 & 0 & 1 \\
\hline 81 & Muedas Taipe Golfer [PE] & & & & & & & & & & & 1 & & 1 & 0 & 1 \\
\hline 82 & Murrugarra Estrada Jeanpier [PE] & & & & & & 1 & & & & & & & 1 & 0 & 1 \\
\hline 83 & Ortiz Salas Carmen Eleana [PE] & & & & & & & & & & & 1 & & 1 & $\mathrm{O}$ & 1 \\
\hline 84 & Pena Camargo Josias Anderson [PE] & & & & & & & 1 & & & & & & 1 & 0 & 1 \\
\hline 85 & Pillpe Meza Raysa Magali [PE] & & & & & & 1 & & & & & & & 1 & $\mathrm{O}$ & 1 \\
\hline 86 & Poma Espinoza Diana [PE] & & & & & & & & & 1 & & & & 1 & 0 & 1 \\
\hline 87 & Quintana Carrillo Wilfredo Dennis [PE] & & & & & & 1 & & & & & & & 1 & 0 & 1 \\
\hline 88 & Quiroz Aguinaga Ulises [PE] & & & & & & & & & & & & 1 & 1 & $\mathrm{O}$ & 1 \\
\hline 89 & Ríos Julcapoma Milton [PE] & & & & & & & & & & & 1 & & 1 & 0 & 1 \\
\hline 90 & Rodrigo Caros Palacios Aguilar [PE] & & & & & & & & & 1 & & & & 1 & 0 & 1 \\
\hline 91 & Román Mendoza Luz Esmeralda [PE] & & & & & & & & 1 & & & & & 1 & 0 & 1 \\
\hline 92 & Rosas Velásquez Diego Fernando [PE] & & & & & & & & & 1 & & & & 1 & 0 & 1 \\
\hline 93 & Salazar Rojas Andree Franklin [PE] & & & & & & & & & 1 & & & & 1 & 0 & 1 \\
\hline 94 & Solís Nuñez Rosa [PE] & & & & & & 1 & & & & & & & 1 & 0 & 1 \\
\hline 95 & Taboada Sotomayor Maria del Pilar [PE] & & & & & & & & & & & 1 & & 1 & $\mathrm{O}$ & 1 \\
\hline 96 & Tapia Quispitupa Betzy Suey [PE] & & & & & & & 1 & & & & & & 1 & 0 & 1 \\
\hline 97 & Teves Rojas Augusto [PE] & & & & & & & & & & & 1 & & 1 & 0 & 1 \\
\hline 98 & Toro Taipicuri Patricia Alexandra [PE] & & & & & & & & & 1 & & & & 1 & 0 & 1 \\
\hline 99 & Torres Aurtua Dora Jesús [PE] & & & & & & & & 1 & & & & & 1 & 0 & 1 \\
\hline 100 & |Uribe Valenzuela Carmen Luisa [PE] & & & & & & & & 1 & & & & & 1 & 0 & 1 \\
\hline 101 & Urquizo Araujo Adan Mak Brayam [PE] & & & & & & & & & & & 1 & & 1 & 0 & 1 \\
\hline 102 & Valderrama Negrón Ana Cecilia [PE] & & & & & & & & & 1 & & & & 1 & 0 & 1 \\
\hline 103 & Zavaleta López Renzo Rafael [PE] & & & & & & & & & 1 & & & & 1 & 0 & 1 \\
\hline 104 & Zorrilla Aliaga Bill Wild [PE] & & & & & & & & & & & 1 & & 1 & $\mathrm{O}$ & 1 \\
\hline & Total & 2 & 4 & 6 & 5 & 22 & 29 & 70 & 71 & 107 & 1 & 21 & 7 & 345 & & \\
\hline
\end{tabular}

Nuevo inventor

Inventor que ya ha publicado 


\section{Tabla 8. Líneas de interés tecnológico}

\begin{tabular}{|c|c|c|c|c|c|c|c|c|c|c|c|c|}
\hline Líneas de interés tecnológico & œ & : & 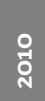 & 总 & กี้ & कू̀ & ఫ্ं & กิ & $\stackrel{\circ}{\circ}$ & 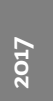 & $\stackrel{\text { స్ }}{\infty}$ & 厄ँّ \\
\hline $\mathrm{A}_{47} \mathrm{C}$ - sillas; sofás; camas. & & & & & 1 & 2 & 3 & 4 & 2 & & & 12 \\
\hline $\begin{array}{l}\text { A63G - tiovivos; columpios; caballos } \\
\text { basculantes; toboganes; montaña rusa; } \\
\text { distracciones publicas análogas. }\end{array}$ & & & & & & 1 & & 4 & 3 & & & 8 \\
\hline $\begin{array}{l}\text { A47B - mesas; escritorios; mobiliario de } \\
\text { oficina; muebles con cajones; cajones; partes } \\
\text { constitutivas generales de los muebles. }\end{array}$ & & 1 & & & 1 & 1 & 1 & 3 & & & & 7 \\
\hline $\begin{array}{l}\text { Do3D - tejidos; métodos de tejido; máquinas } \\
\text { para tejer. }\end{array}$ & & & & & 3 & 1 & & & 2 & 1 & & 7 \\
\hline $\begin{array}{l}\text { Co2F - tratamiento del agua, agua residual, de } \\
\text { alcantarilla o fangos. }\end{array}$ & & & & 2 & 1 & & 1 & & 1 & & 1 & 6 \\
\hline $\begin{array}{l}\text { Eo4B - estructura general de los edificios; } \\
\text { muros, p. Ej. Tabiques; tejados; techos; suelos; } \\
\text { aislamiento y otras protecciones de los edificios. }\end{array}$ & & & & & & 1 & 3 & 2 & & & & 6 \\
\hline $\begin{array}{l}\mathrm{A}_{43} \mathrm{C} \text { - cierres o accesorios para el calzado; } \\
\text { cordones en general. }\end{array}$ & & & & & & & 2 & & 1 & & & 3 \\
\hline $\mathrm{A}_{47} \mathrm{G}$ - utensilios de uso doméstico o de mesa. & & & & & 1 & 1 & & & 1 & & & 3 \\
\hline $\begin{array}{l}\text { A47L - lavado o limpieza domestica; aspiradores } \\
\text { en general. }\end{array}$ & & & & & & & 1 & & 2 & & & 3 \\
\hline A61B - diagnostico; cirugía; identificación. & & 2 & & & & & & & 1 & & & 3 \\
\hline Eo6C - escaleras. & & & & & & & 2 & & 1 & & & 3 \\
\hline $\begin{array}{l}\text { A45B - bastones (ayudas para caminar); } \\
\text { paraguas o sombrillas; abanicos u objetos } \\
\text { similares para señoras. }\end{array}$ & & & & & & & & & 2 & & & 2 \\
\hline $\begin{array}{l}\text { A47J - material de cocina; molinillos de café; } \\
\text { molinillos de especias; aparatos para preparar } \\
\text { las bebidas. }\end{array}$ & & & & & & & 1 & 1 & & & & 2 \\
\hline $\begin{array}{l}\text { A47K - aparatos sanitarios no previstos en otro } \\
\text { lugar; accesorios para el aseo. }\end{array}$ & & & & & 1 & & 1 & & & & & 2 \\
\hline $\begin{array}{l}\text { A63H - juguetes, p. Ej. Trompos, muñecos, aros, } \\
\text { juegos de construcción. }\end{array}$ & & & & & & & & 1 & & 1 & & 2 \\
\hline $\begin{array}{l}\text { BorD - procedimientos o aparatos físicos o } \\
\text { químicos en general - separación. }\end{array}$ & 1 & & & & 1 & & & & & & & 2 \\
\hline $\begin{array}{l}\text { B62B - vehículos propulsados a mano, p. Ej. } \\
\text { Coches de mano o coches de niño; trineos. }\end{array}$ & & & & & & & 2 & & & & & 2 \\
\hline B62D - vehículos de motor; remolques. & & & & & & & & 1 & 1 & & & 2 \\
\hline $\begin{array}{l}\text { B63B - buques u otras embarcaciones flotantes; } \\
\text { equipamiento para embarcaciones. }\end{array}$ & & & & & & & & & 2 & & & 2 \\
\hline $\begin{array}{l}\text { B65F - recogida o retirada de basuras } \\
\text { domesticas o desperdicios similares. }\end{array}$ & & & & & & & 2 & & & & & 2 \\
\hline $\begin{array}{l}\text { Eo4F - trabajos de acabado del edificio, p. Ej. } \\
\text { Escaleras, revestimientos de suelos. }\end{array}$ & & & & & & & & & 2 & & & 2 \\
\hline $\begin{array}{l}\text { F24C - calefacción; hornillas; ventilación. Otras } \\
\text { estufas u hornillas de uso doméstico. }\end{array}$ & & & & & & & 1 & 1 & & & & 2 \\
\hline $\begin{array}{l}\text { Go8B - sistemas de señalización o de llamada; } \\
\text { transmisores telegráficos de ordenes; sistemas } \\
\text { de alarma. }\end{array}$ & & & & & & & & & 1 & & 1 & 2 \\
\hline $\begin{array}{l}\text { A41D - ropa exterior; ropa de protección; } \\
\text { accesorios. }\end{array}$ & & & & & & & & & 1 & & & 1 \\
\hline $\begin{array}{l}\text { A41F - dispositivos de abrochado para prendas } \\
\text { de vestir; artículos de sujeción. }\end{array}$ & & & & & & & & & 1 & & & 1 \\
\hline $\begin{array}{l}\text { A43B - elementos característicos del calzado; } \\
\text { partes constitutivas del calzado. }\end{array}$ & & & & & & & & & 1 & & & 1 \\
\hline
\end{tabular}




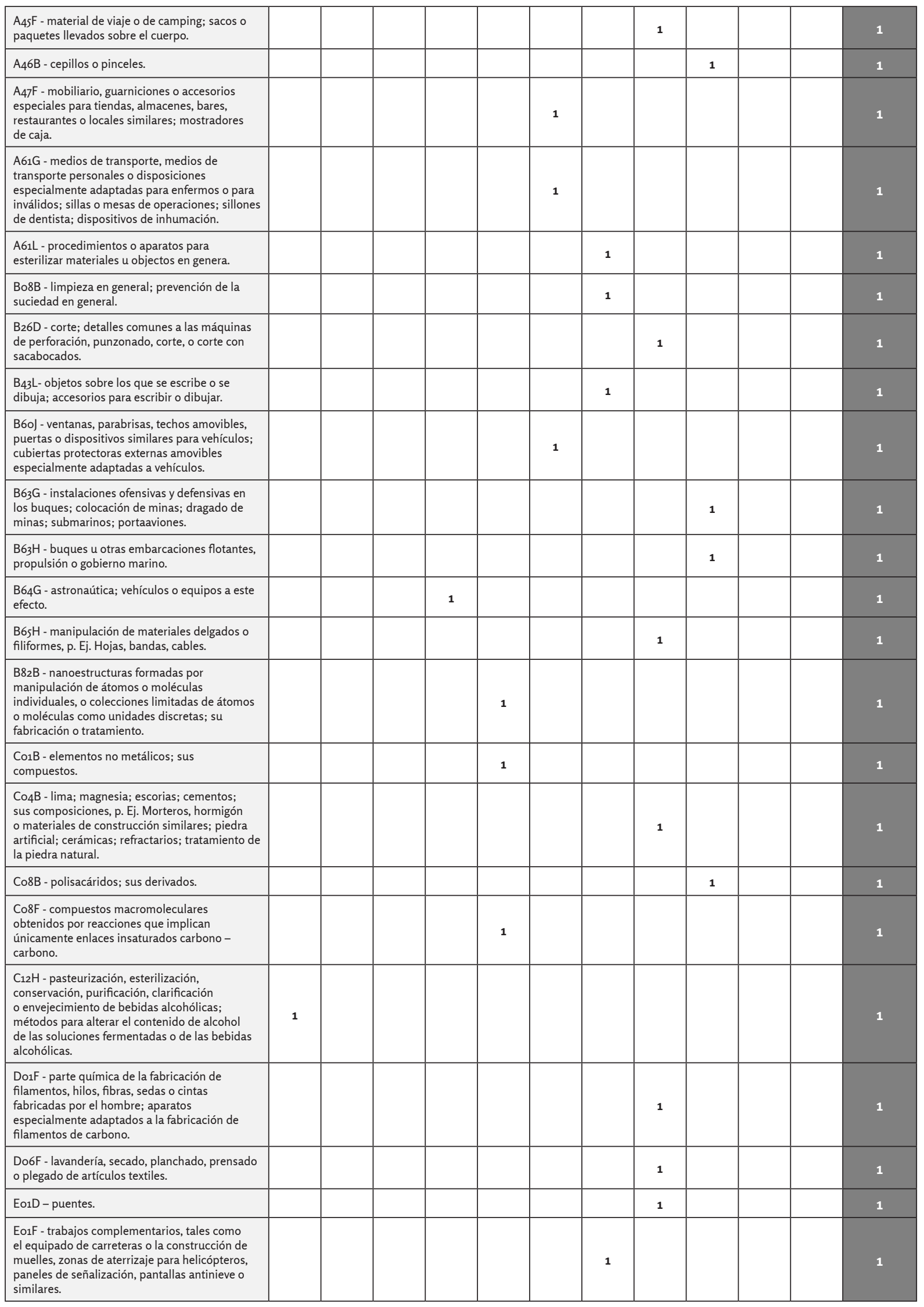




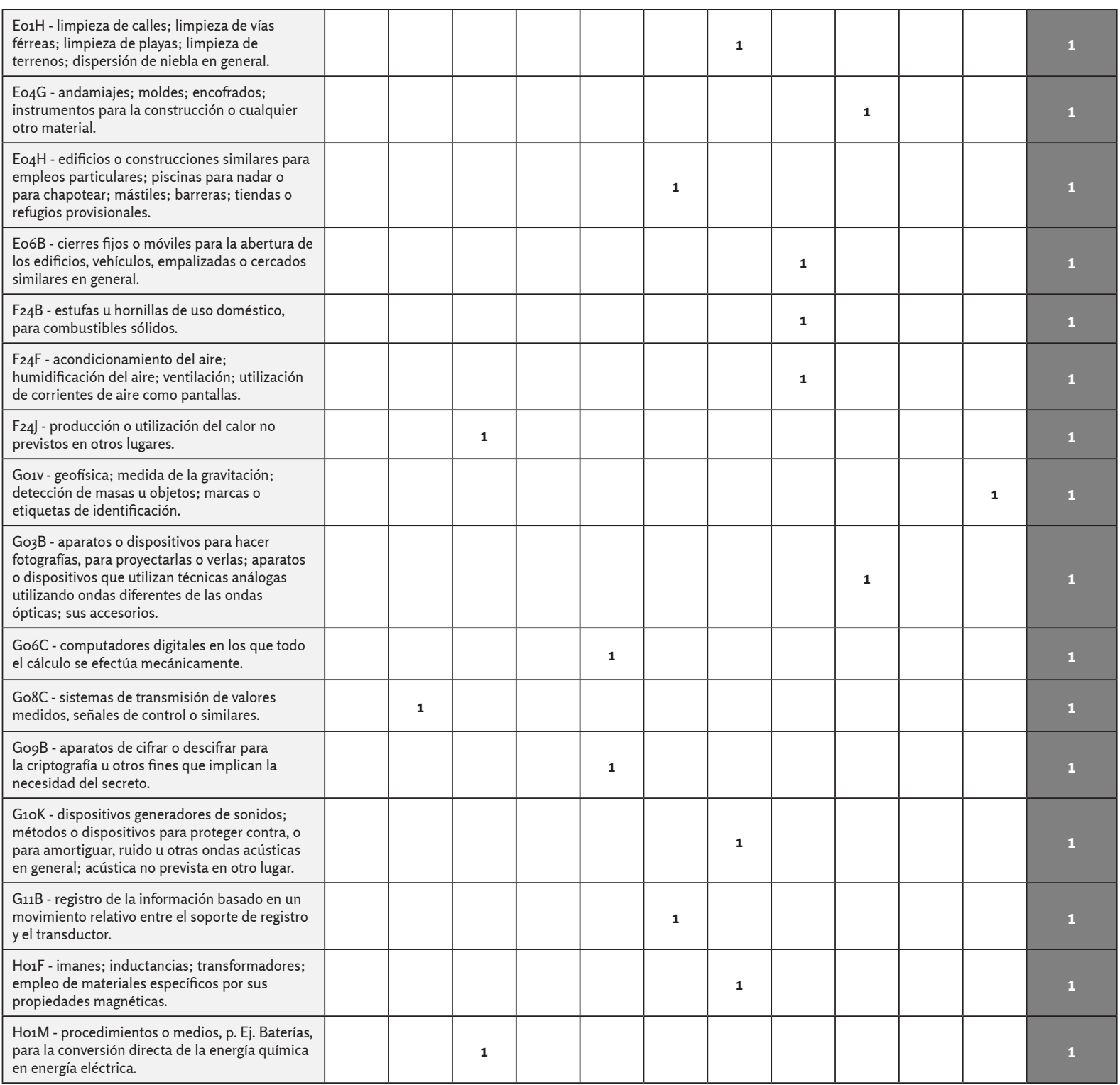




\section{Q Referencias bibliográficas}

» CONCYTEC. 2006. Plan nacional estratégico de ciencia, tecnología e innovación para la competitividad y el desarrollo humano PNCTI 2006 - 2021. Lima: CONCYTEC.

» CONCYTEC. 2014. Estrategia Nacional para el Desarrollo de la Ciencia, Tecnología e Innovación. Crear para crecer. Lima: CONCYTEC.

»Dima, Alina M. y Simona Vasilache.2015. Social network analysis for tacit knowledge management in universities. En Journal of Knowledge Economy. Vol. 6, no. 4, 856-864.

» Ling, JR. 2009. Social network analysis: Theory, method and application. Beijing: Normal University Press.

" Liu, J. 2004. An introduction to school network analysis. Beijing: Social Science Documentation Publishing House.

" Ma, Shao-Qi, Can Jiao y Min-Qiang Zhang. 2011. Application of social network analysis in psychology.En Advances in Psychological Science. Vol. 19, no. 5, 755-764.<http://journal.psych.ac.cn/xlkxjz/EN/Y2011/V19/ 15/755>[Consulta: 29 diciembre 2019].

" Martínez de Ita, María Eugenia, Fernando Julio Piñero y Silvana Andrea Figueroa Delgado. 2013. El papel de la universidad en el desarrollo. Puebla: Benemérita Universidad Autónoma de Puebla; Clacso; Universidad Nacional del Centro de la Provincia de Buenos Aires.<http://biblioteca.clacso.edu.ar/clacso/ se/20140211121020/universidad.pdf>[Consulta: 29 diciembre 2019].

» Ministerio de Educación. 2014. Ley Universitaria. Ley Nº30220. Lima: Ministerio de Educación.

»Molina, José Luis. 2009. Panorama de la investigación en redes sociales. En REDES Revista hispana para el análisis de redes sociales. Vol. 17, no. 11, 235-256.<https://revistes.uab.cat/redes/article/view/v17-n2-molina/383pdf-e-> [Consulta: 29 diciembre 2019].

» Morescalchi, Andrea, Fabio Pammolli, Orion Penner, Alexander M. Petersen y Massimo Riccaboni. 2015. The evolution of networks of innovators within andacross borders: evidence from patent data. En Research Policy. Vol. 44, no. 3,651-668. <https://doi.org/10.1016/j.respol.2014.10.015>

" OECD. 2018The future of education and skills education 2030. Paris: OECD. <https://www.oecd.org/education/2030-project/>

》 OMPI. 2007. Manual de la OMPI de redacción de solicitudes de patente. Ginebra: OMPI. <https://www.wipo. int/publications/es/details.jsp?id=297> [Consulta: 29 diciembre 2019].

»Osca-Lluch, Julia. 2010. Aplicación del análisis de redes al estudio de la investigación española de historia de la ciencia. En REDES - Revista hispana para el análisis de redes sociales. Vol. 19, no. 2, 122-143. <https://doi. org/10.5565/rev/redes.402>

» Paul, Louis C. y Anthony O’LenickJr. 2008. Patent. Peace of mind. New York: Allured Publishing Corporation.

"Rodríguez Ponce, Emilio. 2009. El rol de las universidades en la Sociedad del Conocimiento y en la era de la globalización: evidencia desde Chile. En Interciencia. Vol. 34, no. 11, 824-829. <http://ve.scielo.org/scielo. php?script=sci_arttext\&pid=S0378-18442009001100013> [Consulta: 29 diciembre 2019].

》Russell, Jane M., Ma. Jesús Madera Jaramillo y Shirley Ainsworth. 2009. El análisis de redes en el estudio de la colaboración científica. En REDES- Revista hispana para el análisis de redes sociales. Vol. 17, no. 2, 38-47. $<$ https://doi.org/10.5565/rev/redes.374>

"Stoddart, S. D. R. y Siddiqui, M. K. 2013. Social network analysis of authorship networks and the identification of expert advisors. En Value in Health.Vol. 16, no. 7, 614-614. <https://doi.org/10.1016/j.jval.2013.08.1777>

»SUNEDU. 2018. Informe bienal sobre la realidad universitaria peruana. Lima: SUNEDU. <https://www.sunedu.gob.pe/informe-bienal-sobre-realidad-universitaria/> [Consulta: 29 diciembre 2019]. 
»UNESCO. 2015. Rethinking Education, Towards a global common good? Paris: UNESCO. <https://unesdoc.unesco.org/ark:/48223/pfoooo232555> [Consulta: 29 diciembre 2019].

"UNESCO. 2018. Global education monitoring report 2019. Migration, displacement and education. Building bridges, not walls. Bruselas: UNESCO. <https://unesdoc.unesco.org/ark:/48223/pfoooo265866> [Consulta: 29 diciembre 2019].

"Valente, Thomas W. 1995. Network models of the diffusion of innovations. New Jersey: Hampton Press.

»Villarán de la Puente, Fernando. 2015. Historia de las patentes e invenciones en el Perú. Lima: INDECOPI. 